AN INTERNATIONAL JOURNAL

\title{
Screening of Native Yeast from Agave duranguensis Fermentation for Isoamyl Acetate Production
}

\author{
Gerardo Hernández-Carbajal ${ }^{1}$, Olga Miriam Rutiaga-Quiñones ${ }^{1}$, Araceli Pérez-Silva ${ }^{2}$, \\ Gerardo Saucedo-Castañeda ${ }^{3}$, Adriane Medeiros ${ }^{4}$, Carlos Ricardo Soccol $^{4}$ and Nicolás \\ Óscar Soto-Cruz ${ }^{1 *}$ \\ ${ }^{1}$ Instituto Tecnológico de Durango; Felipe Pesacador 1830 Ote. 34080; Durango - México. ${ }^{2}$ Instituto Tecnológico \\ de Tuxtepec; Víctor Bravo Ahuja s/n, 68350; Tuxtepec, Oxaca. ${ }^{3}$ Universidad Autónoma Metropolitana Iztapalapa; \\ San Rafael Atlixco 186, 09340; México. ${ }^{4}$ Universidade Federal do Paraná; 81531-990; Curitiba - PR - Brasil
}

\begin{abstract}
In this work, fifty yeast strains, isolated from the spontaneous alcoholic fermentation of Agave duranguensis to produce mezcal, were tested using the double coupling system. These yeasts were from the genera Pichia, Torulaspora, Saccharomyces, Kluyveromyces, Deckera, Hanseniaspora, and Candida. P. fermentans ITD00165 was the best isoamyl acetate producer, yielding $0.38 \mathrm{~g} / \mathrm{L}$ of ester after incubation for $24 \mathrm{~h}$, while K. marxianus ITD00211 produced $0.32 \mathrm{~g} / \mathrm{L}$ of ester. Thus P. fermentans ITD00165 could be considered as an excellent choice for use in optimization studies of the culture medium and bioreactor operating conditions to develop a process for biotechnological production of isoamyl acetate.
\end{abstract}

Key words: Banana aroma, Food additive, Native yeast strains

\section{INTRODUCTION}

A vast array of compounds, such as alcohols, esters, fatty acids, and sulphur compounds may be responsible for the flavor of foods (Gratfield. 1988; Dubal et al. 2008; Krings 1998). Food processing can cause a weak aroma in the final product, hence, it is necessary to use the additives (Lemos et al. 2010). Most of these compounds are produced by the chemical synthesis, but a rapid shift to biosynthesis is taking place (Janssens et al. 1992) because consumers have developed a tendency to prefer the food with a "natural" label (Janssens et al. 1992; Lemos et al. 2010). Isoamyl acetate is an ester with great interest in the food industry. It has a consumption of $74,000 \mathrm{~kg}$ per year due to its characteristic banana smell (Torres et al. 2009).

Yeasts produce esters by esterification of alcohols with acetyl co-enzyme A (Verstrepen et al. 2003). Two genes coding for the enzyme alcohol acetyltransferase have been identified in Saccharomyces cerevisiae (Mason and Dufour. 2000). This enzyme catalyzes the reaction between acetyl co-enzyme A and alcohols. Yeasts also produce enzymes with ester hydrolase activity and the balance between these two antagonistic enzyme activities determines the final concentration of isoamyl acetate in the fermentation system (Inoue et al. 1997; Fukuda et al. 1998; Yoshimoto et al. 1999; Rojas et al. 2001). Oda (1996) developed a system for the production of esters, called a doubled coupled system (DCS),

*Author for correspondence: nsoto@itdurango.edu.mx 
which consisted in an immobilized microorganism between two the phases, one solid and one liquid. The hydrophilic solid surface contains the nutrients necessary for the microorganism, while the liquid phase is composed of an organic solvent in which the alcohol to be esterified is dissolved. After alcohol esterification with acetyl co-enzyme $\mathrm{A}$, the resulting ester accumulates in the hydrophobic phase of the system, where it can be quantified and recovered.

Like several studies on alcoholic beverage production (Valero et al., 2002; Abbas. 2006), the objective of this work was to select yeast strains with high capacity of isoamyl acetate production, using the doubled coupled system in order to take advantage of the microbial biodiversity in the region as an alternative to produce a natural banana aroma.

\section{MATERIALS AND METHODS}

\section{Chemicals}

Isoamyl acetate (GC grade), isoamyl alcohol, decane, anhydrous sodium carbonate, potassium sodium tartrate, sodium sulphate, copper sulphate pentahydrate, sulphuric acid, ammonium molybdate, sodium arsenate heptahydrate, and glucose were supplied by Sigma-Aldrich (USA). Peptone, yeast extract, malt extract, and agar were supplied by BD Bioxon (Mexico).

\section{Yeast Strains}

Fifty non-Saccharomyces strains belonging to the genera Candida, Hanseniaspora, Kluyveromyces, Pichia, Torulaspora, and Dekkera, isolated from the spontaneous alcoholic fermentation of Agave duranguensis, were obtained from the Microbial Biotechnology Lab's Culture Collection at the Durango Institute of Technology.

\section{Screening procedure}

The synthesis of isoamyl acetate was carried out following the protocol described by Oda (1996). Two hundred microliters from $24 \mathrm{~h}$ cultures in GPYM medium were spread onto Petri dishes with solid GYMP medium and incubated at $30{ }^{\circ} \mathrm{C}$ for $24 \mathrm{~h}$. After incubation, $8.0 \mathrm{~mL}$ of a $1 \%(\mathrm{v} / \mathrm{v})$ isoamyl alcohol solution in decane was placed onto the agar plate and incubated at $30{ }^{\circ} \mathrm{C}$ for $24 \mathrm{~h}$ with shaking $(100 \mathrm{rpm})$. A negative control was subjected to the same process by adding an isoamyl aceteate in decane solution $(0.3 \mathrm{~g} / \mathrm{L})$ onto
Petri dishes without microorganism. Aliquots of the decane solution were taken after incubation for 6,12 , and $24 \mathrm{~h}$ for analysis by gas chromatography (GC). The GYMP medium contained (g/L): 40 glucose, 5 peptone, 3 yeast extract, 3 malt extract, and 1 magnesium sulfate heptahydrate. The $\mathrm{pH}$ of the medium was adjusted to 6.0.

Isoamyl acetate and isoamyl alcohol concentrations were determined using standard solutions. Measurements reported are the average of three independent plate cultures. Statistical comparisons were made using Analysis of Variance (ANOVA) and the Fisher Least Significant Difference (LSD) post-hoc test with the program Statistica version 7.0 (StatSoft, USA). The concentration of isoamyl acetate was quantified on an Agilent Technologies Network System Gas Chromatograph 6890N (Agilent Technologies, USA) equipped with a flame ionization detector (FID) and an HP-Innowax column (Hewlett-Packard, USA) (length, $30 \mathrm{~m}$; inside diameter, $0.25 \mathrm{~mm}$; film thickness, 0.25 $\mathrm{mm})$. The temperatures of the injector block and detector were 220 and $300{ }^{\circ} \mathrm{C}$, respectively. The oven temperature was programmed as follows: equilibrated at $80^{\circ} \mathrm{C}$ for $10 \mathrm{~min}, 80$ to $150{ }^{\circ} \mathrm{C}$ at $1.5^{\circ} \mathrm{C} / \mathrm{min}$, heated to $170{ }^{\circ} \mathrm{C}$ at $4^{\circ} \mathrm{C} / \mathrm{min}$, heated to $250{ }^{\circ} \mathrm{C}$ at $20{ }^{\circ} \mathrm{C} / \mathrm{min}$, and maintained at $250{ }^{\circ} \mathrm{C}$ for $2 \mathrm{~min}$.

\section{Liquid culture for acetate ester formation}

To measure the ester formation, liquid cultures were grown in $125 \mathrm{~mL}$ flasks containing $50 \mathrm{~mL}$ of sterilized GPYM medium at $\mathrm{pH}$ 6.0. Flasks were incubated at $28^{\circ} \mathrm{C}$ with shaking $(120 \mathrm{rpm})$. Aroma compounds in the culture were measured by the headspace gas chromatography. Isoamyl alcohol $(1 \%)$, sterilized with a $0.2 \mu \mathrm{m}$ filter, was added to flasks at 0 and $12 \mathrm{~h}$ of fermentation to look at its effect on ester production. Yeast cell counts were determined by a Neubauer chamber. Experiments were carried out in triplicate.

Reducing sugar concentrations were measured as described previously (Nelson 1944; Somogyi 1945). Aroma compounds were measured the by headspace analysis of the culture in a gas chromatograph Shimadzu model 17A (Shimadzu corp., Japan), equipped with a flame ionization detector at $230{ }^{\circ} \mathrm{C}$. The operation conditions were as follows: HP-5 capillary column (length, $30 \mathrm{~m}$; inside diameter, $0.32 \mathrm{~mm}$ ), column temperature of $40-150{ }^{\circ} \mathrm{C}$ at a rate of $20^{\circ} \mathrm{C} / \mathrm{min}$, and an injector temperature of $230{ }^{\circ} \mathrm{C}$. Volatile compounds were 
expressed as $\mathrm{ppm} / \mathrm{L}$ of the headspace. External standard compounds were used to identify and quantify the microbial aroma compounds.

\section{RESULTS AND DISCUSSION}

The interest in naturally derived products has grown constantly in the society, but many bioprocesses for bioflavor production are not yet commercially available due to low yields (rarely above $0.1 \mathrm{~g} / \mathrm{L}$ ). Thus, these processes are economically unattractive (Medeiros. 2000; Vandamme. 2002). On the other hand, the advantages of yeast, such as their ease of production, have favored industry to adopt biotechnology as a way to produce bioflavors. Nevertheless, a better understanding of yeast biochemistry and the enzymes involved, fermentation optimization, metabolic regulation, and genetic improvement are primordial to enhance the theyields (Abbas. 2006; Etschmann.
2004; Vandamme. 2002). Studies on ester production by yeast have found that modifying parameters, such as components of the culture media and aeration conditions, have increased aroma production by three times (Etschmann. 2004; Lilly. 2006; Medeiros. 2000; Rojas. 2002)

Except for 13 strains of Torulaspora delbrueckii, all the tested strains were able to produce isoamyl acetate after incubation for $24 \mathrm{~h}$. The tested strains of $T$. delbrueckii could be considered as intermediate producers of isoamyl acetate. However, not all the authors have reported isoamyl acetate as an ester produced by $T$. delbrueckii (Plata et al. 2003; Renault et al. 2009). In this study, some strains had no production of this aroma. Table 1 shows the yeasts in each genus with the highest isoamyl acetate production. According to the statistical analysis, $P$. fermentans ITD00165 was the best isoamyl acetate producer, followed by $K$. marxianus ITD00211 and $T$. delbrueckii ITD00233.

Table 1. Yeast strains with high production of isoamyl acetate after incubation for $24 \mathrm{~h}$.

\begin{tabular}{lc}
\hline Yeast & Isoamyl Acetate* $(\mathbf{g} / \mathbf{L})^{\text {Candida diversa ITD0073 }}$ \\
Candida pseudointermedia ITD00101 & $0.1348 \pm 0.01^{\mathrm{hi}}$ \\
Deckera anomala ITD00015 & $0.1458 \pm 0.02^{\mathrm{hi}}$ \\
Hanseniaspora uvarum ITD00108 & $0.1219 \pm 0.02^{\mathrm{hij}}$ \\
Kluyveromyces marxianus ITD00211 & $0.0822 \pm 0.003^{\mathrm{k}}$ \\
Pichia fermentans ITD00165 & $0.3280 \pm 0.006^{\mathrm{b}}$ \\
Saccharomyces cerevisiae ITD00215 & $0.3829 \pm 0.05^{\mathrm{a}}$ \\
Torulaspora delbruekii ITD00233 & $0.0903 \pm 0.01^{\mathrm{jk}}$ \\
\hline
\end{tabular}

*Means sharing the same letter are not significantly different at confidence level of $95 \%$.

The genus Pichia has been reported previously (Oda and Ohta. 1997; Rojas et al. 2001) as a good ester producer. Oda (1996) reported $P$. heedii and $P$. quercuum as the strains with very good activities in the doubled coupled system for the esterification of aliphatic alcohols and terpenes. Rojas and co-workers (2001) assayed a selection of strains for their ability to esterify the alcohols using the double coupling system. They found that the strain $P$. annomala was able to esterify isoamyl alcohol better than the rest of the strains, providing $3 \mathrm{~g} / \mathrm{L}$ isoamyl acetate. Among the factors responsible for these variations are the genus and species of the strain, the media composition, and the culture conditions (Gatfield. 1988; Verstrepen et al. 2003; Saerens et al. 2008). The production of isoamyl acetate by all the strains of $K$. marxianus tested here agreed with the results reported by
Plata and co-workers (2003), who found that the presence of esters was detected only when the fermented media reached an isoamyl alcohol concentration of $0.01 \mathrm{~g} / \mathrm{L}$. They also found that, at this point, alcohol acetyltransferase was active. These may be signs that the production of isoamyl acetate is determined, at least in part, by the availability of isoamyl alcohol (Fabre et al. 1995; Plata et al. 2003). The strains of Torulaspora have ester hydrolase activity, which is more noticeable in the stationary phase (Renault et al. 2009). The strain ITD00233 showed a slight decrease in the concentration of isoamyl acetate from 0.27 to 0.23 $\mathrm{g} / \mathrm{L}$ during the period from 6 to $12 \mathrm{~h}$, which was minimal compared with a reduction of $50 \%$ reported previously (Plata et al. 2003).

All the strains from the two genera with the highest isoamyl acetate production are compared 
in Figure 1. This figure showed that $P$. fermentans ITD00165, $P$. fermentans ITD00154, and $K$. marxianus ITD00211 were the best producers. Thus, these strains were characterized in terms of their production kinetics.

Figure 2 compares the kinetics of isoamyl acetate production by the two best producing strains $(P$, fermentans ITD00165 and $K$. marxianus ITD00211), as well as one strain with low production ( $P$. fermentans ITD00154). $P$. fermentans ITD00165 had the largest production $(0.39 \mathrm{~g} / \mathrm{L}$ at $24 \mathrm{~h})$ and it produced $75 \%$ of the total isoamyl acetate in $6 \mathrm{~h}$ (Fig. 2A). K. marxianus ITD00211 produced $0.33 \mathrm{~g} / \mathrm{L}$ at $24 \mathrm{~h}$, but produced only $33 \%$ of the total isoamyl acetate in $6 \mathrm{~h}$ (Fig. 2B). P. fermentans ITD00154 had the lowest production $(0.14 \mathrm{~g} / \mathrm{L})$, which was reached at $6 \mathrm{~h}$ (Fig. 2C). In the control experiment without microorganism, the isoamyl acetate concentration remained constant throughout the incubation; nevertheless, the isoamyl alcohol concentration decreased from 0.8 to $0.4 \mathrm{~g} / \mathrm{L}$ after incubation for $6 \mathrm{~h}$ (Fig. 2D). This behavior was very similar to that observed for isoamyl alcohol in the other graphs of this figure, independent of the kinetics of isoamyl acetate production in each graph. These results were strong evidence that isoamyl acetate was the product of yeast metabolism and not due to the esterification of added isoamyl alcohol. Yilmaztekin and co-workers (2009) reported a different behavior for the yeast Williopsis saturnus. They found that isoamyl acetate production by $W$. saturnus was enhanced 3 -fold by the addition of $1 \%$ isoamyl alcohol (as a fusel oil).
These results suggested that $W$. saturnus had a different metabolism than that of P.fermentans reported here, since $W$. saturnus esterified isoamyl alcohol to produce isoamyl acetate.

Isoamyl acetate production by $P$. fermentans ITD00165 was also studied in liquid culture. The maximum ester concentration into the headspace of the culture system was obtained after incubation for $12 \mathrm{~h}$ (Fig. 3). The aroma production reached 33 $\mathrm{ppm} / \mathrm{L}$ with $93.75 \%$ reducing sugar consumption. Figure 1 showed that the isoamyl acetate production observed almost coupled to growth. Once sugar consumption and growth stopped, isoamyl acetate production and its concentration decreased, probably due to loss by evaporation. Rossi and co-workers (2009), also measuring aroma concentration in the headspace of Erlenmeyer flasks, reported similar kinetics of isoamyl acetate production by the fungus Ceratocystis fimbriata in solid substrate fermentation using citric pulp. Another possible contribution to the decrease in product concentration was the action of the enzyme ester hydrolase, since the quantity of ester produced depended on the balance of the activities of alcohol acetyltransferase and ester hydrolase, which differed in each yeast (Lilly. 2006; Bisson. 2010; Garde-Cerdán 2008). For example, the ester hydrolase activity in $P$. heedii CECT 11452 was 28 times higher than in P. anomala CECT 10590, while the alcohol acetyltransferase activity was 17 times higher in $P$. heedii CECT 11452 than in $P$. anomala CECT 10590 (Lilly 2006; Bisson 2010).

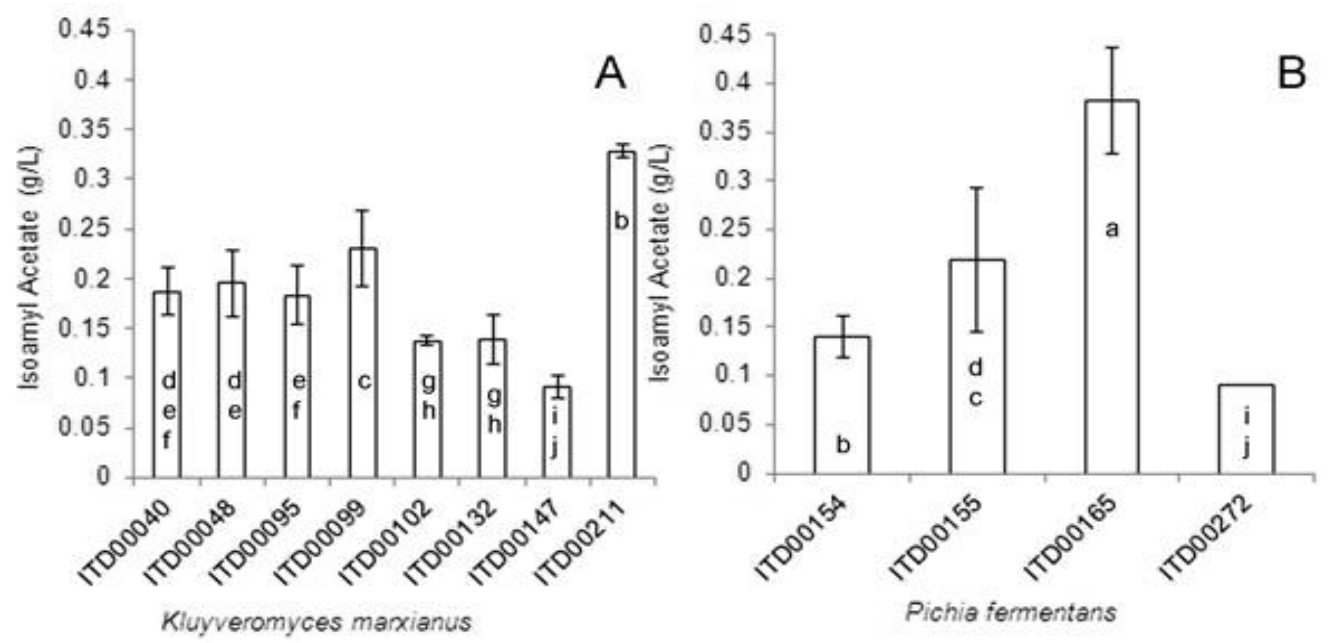

Figure 1 - Isoamyl acetate concentration in the organic phase of the doubled coupled system after incubation for $24 \mathrm{~h}$ with A) Kluyveromyces marxianus and B) Pichia fermentans. 

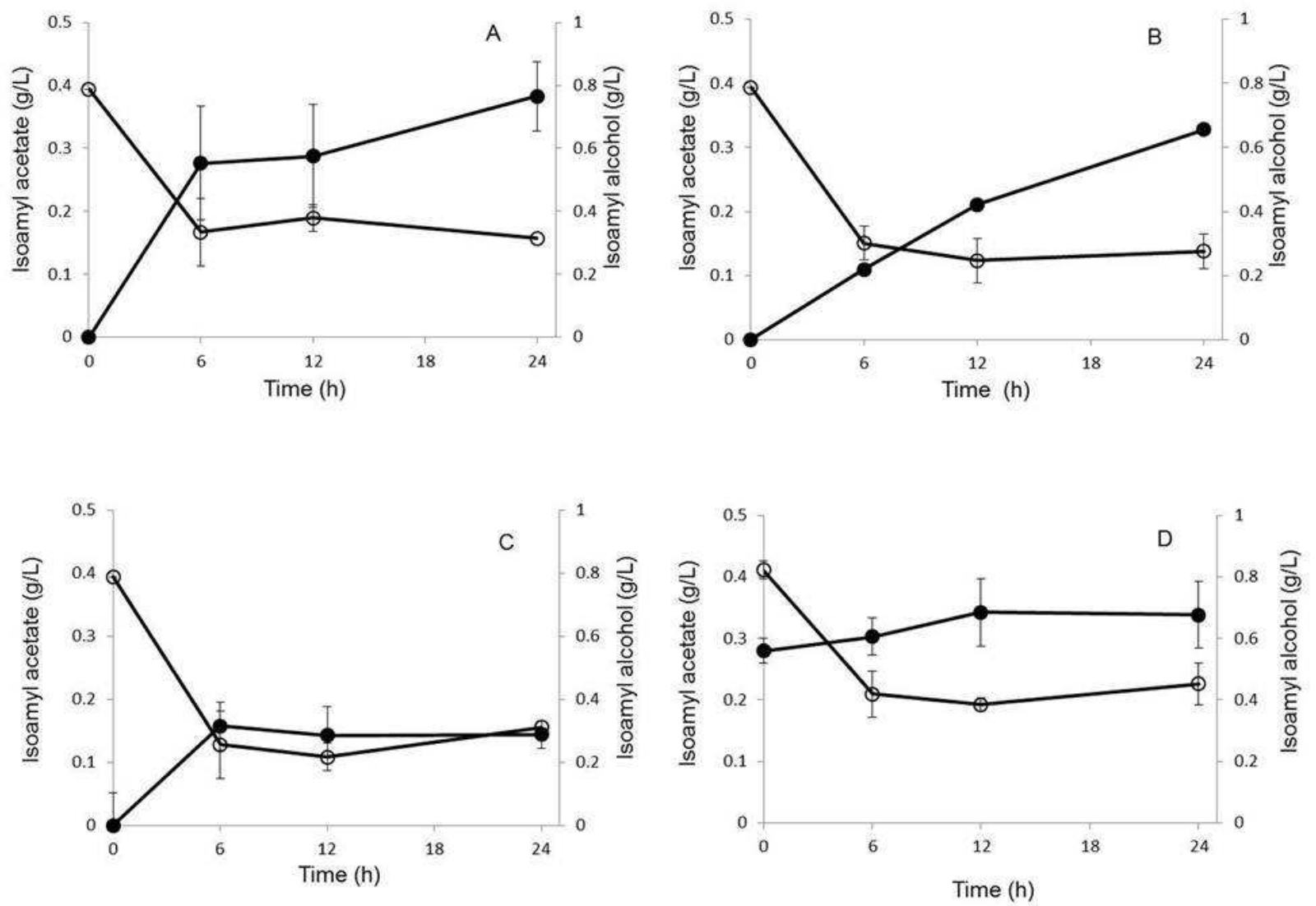

Figure 2 - Production of isoamyl acetate $(\bullet)$ and isoamyl alcohol (o) into decane throughout the incubation. A) Pichia fermentans ITD00165, B) Kluyveromyces marxianus ITD00211, C) Pichia fermentans ITD00154, D) Control without micoorganism. The bars indicate standard deviations for three independent incubations.

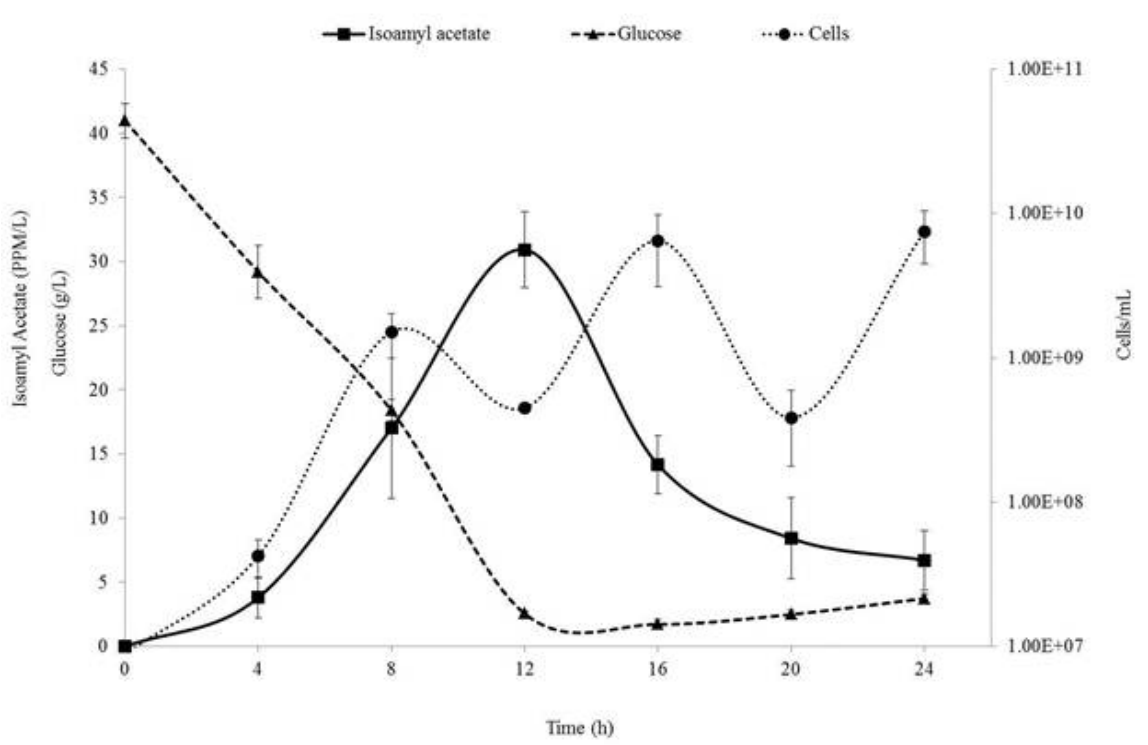

Figure 3 - Production of isoamyl acetate by Pichia fermentans ITD00165 in GYMP medium. Concentrations of biomass $(\mathbf{O})$, isoamyl acetate $(\boldsymbol{Q})$, and reducing sugars $(\boldsymbol{\Lambda})$ ). The bars indicate standard deviations for three independent cultivations. 
Peddie (1990) reported that ester synthesis was very slow at the beginning of fermentation in liquid cultures of $S$. cerevisiae. At this time, oxygen and acetyl CoA were rapidly consumed during the production of unsaturated fatty acids and sterols. Following this, equilibrium between acetyl CoA consumption for fatty acid and sterol synthesis and for ester production was established. Plata and co-workers (2004) found the highest concentrations of isoamyl acetate $(0.15 \mathrm{ppm})$ with a high glucose concentration $(250 \mathrm{~g} / \mathrm{L})$ under semi-anaerobic conditions (150 rpm) and the highest production occurred during the stationary growth phase after $240 \mathrm{~h}$ of the process. Rojas and co-workers (2001) reported that the highest isoamyl acetate concentration $(10.21 \mathrm{ppm})$ was found in $P$. anomala cultures grown with glucose $(40 \mathrm{~g} / \mathrm{L})$ under semi-anaerobic $(120 \mathrm{rpm})$ conditions, which was approximately 19-fold higher than that produced by $S$. cerevisiae cultures after $48 \mathrm{~h}$ of the process. In contrast, in the present study, the synthesis of isoamyl acetate occurred during the exponential growth phase (after growth for $4-8 \mathrm{~h})$, peaked at $12 \mathrm{~h}(32 \mathrm{ppm} / \mathrm{L})$ when the stationary phase was reached, and then began to decline (Fig. 3). There was a 95\% consumption of the initial reducing sugars during the first $12 \mathrm{~h}$ of incubation. Subsequently, there was an increase of about 5\% during the following $12 \mathrm{~h}$ incubation. This could be due to the presence of invertase, which could be hydrolyzing the residual sucrose from the malt extract present in the culture medium. This increase was not considered significant, and in any case did not impact the production of isoamyl acetate.

\section{CONCLUSION}

The doubled coupled system permitted to identify the potential of non-Saccharomyces yeasts, isolated from the alcoholic fermentation of agave to produce acetate esters. In particular, the strain $P$. fermentans ITD00165 was an excellent choice for use in optimization studies of the culture medium and bioreactor operating conditions to develop a process for biotechnological production of isoamyl acetate.

\section{ACKNOWLEDGEMENTS}

The authors thank the financial support from "Programa de Mejoramiento del Profesorado" (project "Red Temática de Colaboración Académica MICROBIOMEX - Microbiología y Biotecnología de Cepas Mexicanas Fúngicas de Interés Industrial"). The first author also acknowledges the scholarship from CONACyT.

\section{REFERENCES}

Abbas AC. Production of Antioxidants, Aromas, Colours, Flavours, and Vitamins by Yeasts. In: Yeasts in Food and Beverages. 1st ed. Amparo Querol, Graham H. Fleet, Eds., Springer-Verlag Berlin Heidelberg; 2006. p. 285 - 334.

Bisson LF, Karpel, JE. Genetics of Yeast Impacting Wine Quality. Annual Review of Food Science and Technology.2010; 1,139-162

Dubal AS, Tilkari YP. Momin SA. Borkar IV. Biotechnological routes in flavour industries. Advanced Biotech. 2008; 6(08): 20-31.

Etschmann MMW, Sell D, Schrader J. Medium optimization for the production of the aroma compound 2-phenylethanol using a genetic algorithm., J mol catal B enzyme. 2004; 29. 187-193

Fabre CE, Duvian V J, Blanc PJ, Goma, G. Identification of volatile flavour compounds obtained in culture of Kluyveromyces marxianus, Biotechnol Lett. 1995; 17, 1207-1212.

Fukuda K., Yamamoto A, Kiyokawa Y, Yanagiuchi T, Wakai Y, Kitamoto K., Inoue Y, et al. Balance of Activities of Alcohol Acetyltransferase and Esterase in Saccharomyces cerevisiae Is Important for Production of Isoamyl Acetate, Appl. Environ. Microbiol, 1998; 64. 4076-4078.

Garde Cerdán T, Ancín Azpilicueta C. Effect of the addition of different quantities of amino acids to nitrogen-deficient must on the formation of esters, alcohols, and acids during wine alcoholic fermentation, SGLWT/SOSSTA. 2008; 41. 501-510

Gatfield IL. Production of flavour and aroma compounds by biotechnology. Food Technol. 1988; 42(10): 110-122.

Hernández Orte P, Cersosimo M, Loscos N, Cacho J, GarciaMoruno E, Ferreira V. The development of varietal aroma from non-floral grapes by yeasts of different genera. Food Chem. 2008: 107. 1064-1077.

Inoue Y, Trevanichi T, Fukuda K., Izawa S, Wakai Y, Kimura K.. Roles of Esterase and Alcohol Acetyltransferase on Production of Isoamyl Acetate in Hansenula mrakii. J. Agric. Food Chem. 1997; 45(3): 644-649 
Janssens L, De Pooter HL, Schamp NM, Vandamme EJ. Production of flavors by microorganisms. Proc. Biochem. 1992; 27: 195-215.

Krings U, Berger RG. Biotechnological production of flavours and fragrances. Appl. Microbiol. Biotechnol. 1998; 49(1): 1- 18.

Lemos J, Sililva J, Dionísio A, Pastore G. Biotechnological production of bioflavors and functional sugars. Ciênc. Tecnol. Aliment. 2010; 30. 7-18

Lilly M, Bauer FF, Lambrechts MG, Swiegers JH, Cozzolino D, Pretorius SI. The effect of increased yeast alcohol acetyltransferase and esterase activity on the flavour profiles of wine and distillates. Yeast, 2006; 23. 641-659.

Mason AB, Dufour JP. Alcohol acetyltransferases and the signicance of ester synthesis in yeast. Yeast, 2000; 16(14): 1287-1298.

Miller GL, Blum R, Glennon WE, Burton AL. Measurement of carboxymethylcellulase activity. Anal. Biochem. 1960; 2:127-132

Nelson N. A photometric adaptation of the Somogyi method for the determination of glucose. J. Biol. Chem. 1944; 153: 375-380.

Oda S. Coupled Process of saccharide fermentation and microbial esterification. U.S. patent 5,780,275 September 1996

Oda S, Ohta H. Double coupling of acetyl coenzyme A production and microbial esterification with alcohol acetyltransferase in an interface bioreactor. $J$. Ferment. Bioeng. 1997; 83(5): 423-428.

Medeiros A, Pandey A Freitas JS, Christen P, Soccol CR. Optimization of the production of aroma compounds by Kluyveromyces marxianus in solidstate fermentation using factorial design and response surface methodology. Biochem. Eng. J. 2000; 6. 3339

Peddie HAB. Ester formation in brewery fermentations. J. Inst. Brew. 1990; 96: 327-331.

Plata C, Millan C, Mauricio JC, Ortega JM. Formation of ethyl acetate and isoamyl acetate by various species of wine yeasts. Food Microbiol. 2003; 20. 217-224

Renault PC, Miot Sertier P, Marullo P, Hernádez Orte L, Lagarrigue A, Lonvaud F, et al. Genetic characterization and phenotypic variability in Torulaspora delbrueckii species: potential applications in the wine industry. Int $J$ Food Microbiol. 2009; 134(3): 201-210.

Rojas V, Gil JV, Piñaga F, Manzanares P. Studies on acetate ester production by non-Saccharomyces wine yeasts. Int J Food Microbiol. 2001; 70. 283-289.
Rojas V, Gil JV, Piñaga F, Manzanares P, Gavara R, Flors A. Measurement of alcohol acetyltransferase and ester hydrolase activities in yeast extracts. Enzyme Microb Tech. 2002; 30. 224-230.

Saerens SM, Delvaux F, Verstrepen K.J, Van Dijck P, Thevelein JM, Delvaux F R. Parameters Affecting Ethyl Ester Production by Saccharomyces cerevisiae during Fermentation. Appl. Environ. Microbiol.. 2008; 74. 454-461.

Somogyi M. A new reagent for the determination of sugars. J. Biol. Chem. 1945; 160: 61-687.

Torres S, Baigorí MD, Swathy SL, Pandey A, Castro GR. Enzymatic synthesis of banana flavour (isoamyl acetate) by Bacillus licheniformis S-86 esterase. Food Res Int. 2009; 42. 454-460.

Valero E, Moyano L, Millan MC, Medina M, Ortega JM. Higher alcohols and esters production by Saccharomyces cerevisiae. Influence of the initial oxygenation of the grape must. Food Chem. 2002: 78. 57-61

Vandamme EJ, Soetaert W. Bioflavours and fragrances via fermentation and biocatalysis. J. Chem. Technol. Biotechnol. 2002; 77. 1323-1332.

Verstrepen KJ, Derdelinckx JP, Dufour J, Winderickx JM, Thevelein IS, Pretorius, Delvaux FR. Flavoractive esters: adding fruitiness to beer. J. Biosci. Bioeng. 2003; 96. 110-118.

Yilmaztekin M, Erten H, Cabaroglu T. Enhanced production of isoamyl acetate from beet molasses with addition of fusel oil by Williopsis saturnus var. saturnus. Food Chem. 2009;112. 290-294.

Vandamme EJ, Soetaert W. Bioflavours and fragrances via fermentation and biocatalysis. J. Chem. Technol. Biotechnol. 2002; 77. 1323-1332.

Yoshimoto H, Fujiwara D, Momma T, Tanaka K, Sone $\mathrm{H}$, Nagasawa N, Tamai, Y. Isolation and characterization of the ATF2 gene encoding alcohol acetyltransferase II in the bottom fermenting yeast Saccharomyces pastorianus. Yeast. 1999; 15(5):409417.

Received: May 02, 2012; Revised: December10, 2012; Accepted: May 16, 2013. 


\section{PAGINA EM BRANCO}

